\title{
No evidence for inbreeding avoidance in a natural population of song sparrows (Melospiza melodia)
}

\author{
Keller, L F ; Arcese, P
}

\begin{abstract}
We studied mate choice and inbreeding avoidance in a natural population of song sparrows (Melospiza melodia) on Mandarte Island, Canada. Inbreeding occurred regularly: 59\% of all matings were between known relatives. We tested for inbreeding avoidance by comparing the observed levels of inbreeding to those expected if mate choice had been random with respect to relatedness. Independent of our assumptions about the availability of mates in the random mating model, we found that the expected and observed distributions of inbreeding coefficients were similar. as was the expected and observed frequency of close (f greater than or equal to 0.125) inbreeding. Furthermore, there was no difference in relatedness of observed pairs and those that would have resulted had birds mated instead with their nearest neighbors. The only evidence to suggest any inbreeding avoidance was a reduced rate of parentoffspring matings as compared to one random mating model but not the other. Hence, despite substantial inbreeding depression in this population, we found little evidence for inbreeding avoidance through mate choice. We present a simple model to suggest that variation in inbreeding avoidance behaviors in birds may arise from differences in survival rates: in species with low survival rates, the costs of forfeiting matings to avoid inbreeding may exceed the costs of inbreeding.
\end{abstract}

DOI: https://doi.org/10.1086/286176

Posted at the Zurich Open Repository and Archive, University of Zurich

ZORA URL: https://doi.org/10.5167/uzh-53665

Journal Article

Published Version

Originally published at:

Keller, L F; Arcese, P (1998). No evidence for inbreeding avoidance in a natural population of song sparrows (Melospiza melodia). American Naturalist, 152(3):380-392.

DOI: https://doi.org/10.1086/286176 


\title{
No Evidence for Inbreeding Avoidance in a Natural Population of Song Sparrows (Melospiza melodia)
}

\author{
Lukas F. Keller* and Peter Arcese $\dagger$
}

Department of Wildlife Ecology, 1630 Linden Drive, University of Wisconsin-Madison, Madison, Wisconsin 53706

Submitted November 25, 1997; Accepted February 18, 1998

AвSTRACT: We studied mate choice and inbreeding avoidance in a natural population of song sparrows (Melospiza melodia) on Mandarte Island, Canada. Inbreeding occurred regularly: 59\% of all matings were between known relatives. We tested for inbreeding avoidance by comparing the observed levels of inbreeding to those expected if mate choice had been random with respect to relatedness. Independent of our assumptions about the availability of mates in the random mating model, we found that the expected and observed distributions of inbreeding coefficients were similar, as was the expected and observed frequency of close $(f \geq 0.125)$ inbreeding. Furthermore, there was no difference in relatedness of observed pairs and those that would have resulted had birds mated instead with their nearest neighbors. The only evidence to suggest any inbreeding avoidance was a reduced rate of parent-offspring matings as compared to one random mating model but not the other. Hence, despite substantial inbreeding depression in this population, we found little evidence for inbreeding avoidance through mate choice. We present a simple model to suggest that variation in inbreeding avoidance behaviors in birds may arise from differences in survival rates: in species with low survival rates, the costs of forfeiting matings to avoid inbreeding may exceed the costs of inbreeding.

Keywords: F statistics, inbreeding, inbreeding avoidance, kinship, pedigree, random mating.

The progeny of outbred matings are often more fit than those of inbred matings, and thus natural selection should favor behaviors that reduce the occurrence of inbreeding (e.g., Darwin 1876; Fisher 1949). In particular, the fitness costs of inbreeding, referred to collectively as "inbreeding depression," are assumed to be a driving

* Present address: Department of Ecology and Evolutionary Biology, Princeton University, Princeton, New Jersey 08544-1003; E-mail: lfkeller@princeton.edu.

†E-mail: arcese@calshp.cals.wisc.edu.

Am. Nat. 1998. Vol. 152, pp. 380-392. (c) 1998 by The University of Chicago. 0003-0147/98/5203-0005\$03.00. All rights reserved. force in the evolution of mating and dispersal systems (e.g., Johnson and Gaines 1990; Weatherhead and Forbes 1994).

Inbreeding depression has been widely demonstrated in captive populations (e.g., Wright 1977; Charlesworth and Charlesworth 1987; Ralls et al. 1988). Clear evidence for inbreeding depression in wild populations of animals has been harder to come by, but several recent studies have shown that inbreeding can reduce fitness substantially under natural conditions (e.g., Chen 1993; Jiménez et al. 1994; Keller et al. 1994; Keller 1998). Taken together, these suggest that reductions in fitness associated with inbreeding often are severe enough that mechanisms to facilitate inbreeding avoidance could have evolved (e.g., Jiménez et al. 1994; Pusey and Wolf 1996). Thus, it is commonly assumed that most organisms tend to avoid close inbreeding (e.g., Hamilton 1987).

For the evolution of inbreeding avoidance behaviors to occur, several conditions must be met (see, e.g., Waser et al. 1986). One is that the costs of inbreeding must exceed the costs of avoiding inbreeding. However, data on inbreeding avoidance and its costs in nature are rare (Johnson and Gaines 1990; Pärt 1996; but see Koenig et al. 1998). Harvey and Ralls (1986) summarized the available data and concluded that mammals and birds generally avoid mating with close relatives. Pusey and Wolf (1996) concluded that evidence for adaptations to avoid close inbreeding in animals is accumulating, but all of these authors emphasized the need for more data before any generalizations can be made.

Behaviors that lead to inbreeding avoidance can be grouped into one of two general categories: those that act via dispersal to separate relatives spatially and reduce the probability of mating with kin (e.g., Pusey 1987); and those that allow animals that reside with relatives to avoid mating with them (e.g., Blouin and Blouin 1988). Sex-biased dispersal is the main mechanism of the first category; mechanisms in the latter category include the suppression of reproduction in offspring in the presence of related adults and the active choice of nonkin as mates (e.g., Blouin and Blouin 1988). 
Dispersal as a mechanism of inbreeding avoidance in birds and mammals has received considerable attention (see, e.g., Pusey 1987 and Johnson and Gaines 1990 for reviews). However, fewer studies have addressed the second category of inbreeding avoidance behaviors. In this study, we ask if inbreeding is actively avoided through mate choice in a population of song sparrows (Melospiza melodia) resident on Mandarte Island, British Columbia. Earlier work on Mandarte Island suggests similar dispersal distances for male and female song sparrows on the island (Tompa 1964; Arcese 1989b), and the distance dispersed on Mandarte Island is not related to the probability of settling with a relative (Arcese 1989b). Given these dispersal patterns and the isolation and small size of the Mandarte population (median number of breeding birds $=89$ ), inbreeding should occur regularly in the absence of active mate choice to avoid inbreeding. In addition, inbreeding depression in song sparrows on Mandarte Island is substantial: eggs produced by full-sib matings $(f=0.25)$ suffer a $49 \%$ reduction in survival to breeding age on average (Keller 1998). Thus, individuals that avoid mating with relatives might be expected to benefit substantially by doing so.

We first present data showing that inbreeding indeed occurs regularly on Mandarte; we then test for evidence of inbreeding avoidance via mate choice by comparing observed levels of inbreeding to those expected under three different models of random mating. Finally, we present a simple model to suggest that adult survival rates may be an important factor modulating the costs of inbreeding avoidance and thus the presence or absence of inbreeding avoidance behaviors in a species.

\section{Approaches to the Analysis of Inbreeding Avoidance by Mate Choice}

Two main approaches have been employed to study inbreeding avoidance through mate choice. The first has been to observe mate choice in experiments, where individuals choose from a variety of potential mates that vary in their degree of relatedness (review in Pusey and Wolf 1996). This approach allows experimenters to control for potentially confounding variables (familiarity, e.g.) and to assess whether kin recognition influences mate choice. However, it is generally difficult to generalize from laboratory conditions used in such experiments to the conditions experienced by wild-living animals (e.g., Bateson 1983). Moreover, this approach does not allow an assessment of how ecological factors-such as competition for territories, the costs of dispersal, or the survival rates of territory holders-may constrain mate choice and thus inbreeding avoidance in nature (e.g., Waser et al. 1986; Weatherhead and Boak 1986; Arcese 1989a, 1989b).
The second approach has been to compare the observed level of inbreeding in populations to that expected under the assumption of random mating in a finite population (e.g., van Noordwijk et al. 1985; van Tienderen and van Noordwijk 1988; Gibbs and Grant 1989; Hoogland 1992). This approach allows researchers to assess the degree to which inbreeding avoidance occurs in nature, but it lacks experimental control of potentially confounding variables. Moreover, the departure of observed from expected levels of inbreeding depend on the assumptions of the null model, especially with regard to which individuals are assumed to be available as potential mates (e.g., Ralls et al. 1986; van Tienderen and van Noordwijk 1988; Pärt 1996).

We employed the second approach to test if inbreeding was avoided by relatives in our study populations. We expected to find fewer inbred matings than predicted under a random null model. To create the null models, we defined the set of potential mates under three alternative assumptions about breeding dispersal, mate switching, and recruitment of new breeders to the population. We surmised that if our results obtained by comparing our observations with the expectations under each null model were similar, our results could be considered robust with respect to a range of assumptions about the availability of mates in our study population.

\section{Methods}

\section{Study Site and Species}

Mandarte Island is a 6-ha islet in Haro Strait, British Columbia, Canada. Several islands with resident song sparrow populations lie within 1.2-6 km. Details of Mandarte Island are given by Tompa (1964) and Drent et al. (1964). Song sparrows on Mandarte show strong natal philopatry, and very few birds immigrate to the population on average $(1.18 / \mathrm{yr}, N=18 \mathrm{yr})$. Males and females show strong breeding philopatry within the island, typically breeding in the same territory year after year. Smith (1981) describes the general field methods employed on Mandarte. In brief, starting in 1975 each territory was visited every 5-7 d from about March-July each year, and the mating status of the territorial male and female was determined (see Arcese 1989b). Nests were located by observing females building the nests or taking breaks from incubation. Regular checks were made subsequently to determine if nests failed, to color-band nestlings individually, and to quantify nestling survival. Adults were individually marked starting in 1974. In the analyses presented here, a male and female were defined as a mated pair if they initiated at least one nesting attempt. Because Mandarte is a small island covered by grass and shrubs, with the shrub patches well divided by trails, and because 


\section{The American Naturalist}

territorial males are conspicuous during the breeding season, we were able to locate all breeding pairs each year.

\section{Inbreeding Estimates}

We determined the parents of all broods through detailed observations of parental behavior and used this information to construct a pedigree spanning 16 generations from 1975 through 1995. From this pedigree we calculated Wright's coefficient of inbreeding $f$ (Wright 1969) for each pair using PEDSYS (Southwest Foundation for Biomedical Research 1998), the Stevens-Boyce algorithm (Boyce 1983), and the KINSHIP option. More details of pedigree construction and calculations of inbreeding coefficients are given elsewhere (Keller 1998). Because pedigrees are shallow during the first years of a study, and inbreeding coefficients therefore less meaningful for this period, we restricted the present analysis to the years 1981-1995.

Undetected extrapair fertilizations can lead to inaccurate assignments of parentage. Preliminary results from DNA analyses suggest that about $15 \%$ of young on Mandarte are sired by extrapair males (Keller 1996). Hence, the majority of observed matings were also the realized matings, and the pair male sired at least one offspring in $97 \%$ of all matings analyzed. However, the extrapair fertilizations introduce error variance in estimates of the inbreeding coefficients, and this could bias our results if extrapair matings were biased with respect to kinship. There was, however, no correlation between the likelihood of a female engaging in extrapair matings and the degree of relatedness between her and her mate $(N=$ 64 pairs, $P \geq .82$; Keller 1996). Therefore, while the extrapair fertilizations introduced error variance in our estimates of inbreeding, they are unlikely to have biased them.

\section{Expected Inbreeding under Random Mating}

We calculated the expected levels of inbreeding under random mating using three alternative sets of assumptions about breeding dispersal, mate switching, and recruitment of new breeders to the population. In the first set, we assumed that all pairs in any year were formed from all surviving adults and recruits from the prior year without regard to their prior mating status. We will refer to this set of potential mates in the following as "All Mates." In the second set, we assumed that only those birds breeding for the first time and those birds whose mate failed to survive to the subsequent breeding season were available to mate. This second assumption accom- modates the notion that birds whose prior mate was still alive may have been unavailable for remating. Gibbs and Grant (1989) used this approach to analyze inbreeding avoidance in medium ground finches (Geospiza fortis). We denote this set of potential mates as "New Mates." Finally, in the third set we assumed that those birds that nested in the neighboring territory nearest to the focal pair were potential mates. This last approach differs from the first two in that it allows for unknown constraints imposed on potential matings by population structure and dispersal patterns (van Noordwijk et al. 1985; van Tienderen and van Noordwijk 1988). It seems reasonable to assume that neighbors are potential mating partners during pair formation.

For All Mates and New Mates, we "paired" every available male with every available female in each year and calculated the inbreeding coefficient that would have resulted from that pairing. This procedure is equivalent to mating each member of one sex with a randomly chosen member of the other sex and repeating this procedure many times (e.g., Gibbs and Grant 1989). By doing so we obtained a distribution of inbreeding coefficients under the assumption of random mating among the available mates, which we then compared to the observed levels of inbreeding among the actual pairs. For each year separately, we compared the mean of the two distributions (Mann-Whitney $U$-test) as well as the distributions as a whole (Kolmogorov-Smirnov two-sample test). In addition, we compared the expected to the observed proportion of matings with $f \geq 0.125$ (half-sibs or more closely related matings) with a $G$-test. We performed this last comparison because population-wide comparisons of inbreeding could mask subtle effects of relatedness. For example, avoidance of close kin as mates could be offset by preferential mating with more distant kin (see, e.g., Bateson 1978).

For the nearest neighbor data, we calculated the inbreeding coefficients that would have resulted if the male and the female of the observed pair had mated with the neighboring female and male, respectively. Hence, two inbreeding coefficients were calculated for each pairneighbor comparison. We averaged these two inbreeding coefficients, subtracted this average from the inbreeding coefficient of the observed pair, and calculated the mean of all these differences for each year. The lack of a difference in the mean relatedness of observed pairs and neighbors indicates that individuals neither avoided nor preferred relatives as mates. We tested the hypothesis that the differences did not differ from 0 in each year using a Wilcoxon signed-rank test.

Because all of these analyses were repeated for each year, we used the standard Bonferroni correction to keep 
the table-wide Type I error at 0.05 . Using the less conservative sequential instead of the standard Bonferroni technique (Rice 1989) did not change any of the conclusions.

\section{Power Analyses}

We performed power analyses for the G-tests, the MannWhitney $U$-tests, and the Wilcoxon signed-rank tests. Power of the $G$-tests was calculated analytically following O'Brien and Muller (1993). Power analyses for nonparametric statistical tests require a Monte Carlo approach. We calculated the power of the Mann-Whitney $U$-tests in our application as follows: using certain rules of inbreeding avoidance (e.g., no matings with $f \geq 0.25$ ), we created a data set of "observed" inbreeding coefficients for a particular year by randomly sampling the appropriate number of matings from the set of all possible matings (All Mates). Any mating that was excluded by the chosen inbreeding avoidance rule was rejected and another mating was drawn. The mean inbreeding coefficient obtained from this artificial data set of "observed" matings was then compared with the mean inbreeding coefficient expected under random mating with a Mann-Whitney $U$-test, and the significance level of the test was recorded. We repeated this process 1,000 times. The power of each individual $U$-test was calculated as the proportion of statistically significant tests $(P \leq .0033$ after Bonferroni correction) and the tablewide power as the power of the most powerful individual $U$-test. Power for the Wilcoxon signed-rank tests was calculated in a similar fashion, but each randomly chosen pair was matched with the nearest neighbor and the difference in resulting inbreeding coefficients was calculated as outlined above.

The power analyses for the $G$-test were performed with the UnifyPow module for SAS produced by R. O'Brien (O'Brien and Muller 1993). All statistical comparisons were performed with SAS version 6.07 (SAS Institute 1990).

\section{Wright's F Statistics}

Wright (summarized in 1965) showed that observed and expected levels of inbreeding in a pedigreed population and the degree of inbreeding avoidance or preference could be represented using his $F$ statistics. This approach is based on Wright's original derivation of the $F$ statistics as correlation coefficients rather than on the now more familiar representation as ratios of variances in gene frequencies. Crow and Kimura (1970, p. 107) show that the two are equivalent. We chose to represent our results in terms of Wright's $F$ statistics because they are wellknown quantities and because they allow comparisons between studies based on pedigrees and molecular data. In the context of a single pedigreed population, the population at any period during the study is treated as a subpopulation of the total of the infinite number of possible populations that might have been derived from the founding population (Wright 1965, 1969). Following this approach, the observed mean inbreeding coefficient is equal to $F_{\mathrm{IT}}$, while $F_{\mathrm{ST}}$ quantifies the mean inbreeding coefficient expected under random mating. In other words, $F_{\mathrm{ST}}$ is the correlation between random gametes within the pedigreed population relative to gametes of the total of all possible populations. In contrast, $F_{\mathrm{IS}}$ represents the degree of nonrandom mating - that is, inbreeding avoidance or preference- and can be calculated from the formula $F_{\mathrm{IS}}=\left(F_{\mathrm{IT}}-F_{\mathrm{ST}}\right) /\left(1-F_{\mathrm{ST}}\right) ; F_{\mathrm{IS}}$ is negative when $F_{\mathrm{ST}}$ is greater than $F_{\mathrm{IT}}$, that is, when there is inbreeding avoidance.

\section{Results}

\section{Occurrence of Inbreeding}

Inbreeding occurred regularly on Mandarte Island. Overall, inbreeding coefficients ranged from $f=0$ to $f=$ 0.305 (fig. 1). Fifty-nine percent of 479 matings occurring after 1981 were among known relatives $(f>0)$, and 18 matings $(3.8 \%)$ were between full-sibs or parents and their offspring. Of those 18 matings, 10 were between full-sibs that had been raised in different nests, six between full-sibs that had been raised together in the same nest, and two were mother-son matings.

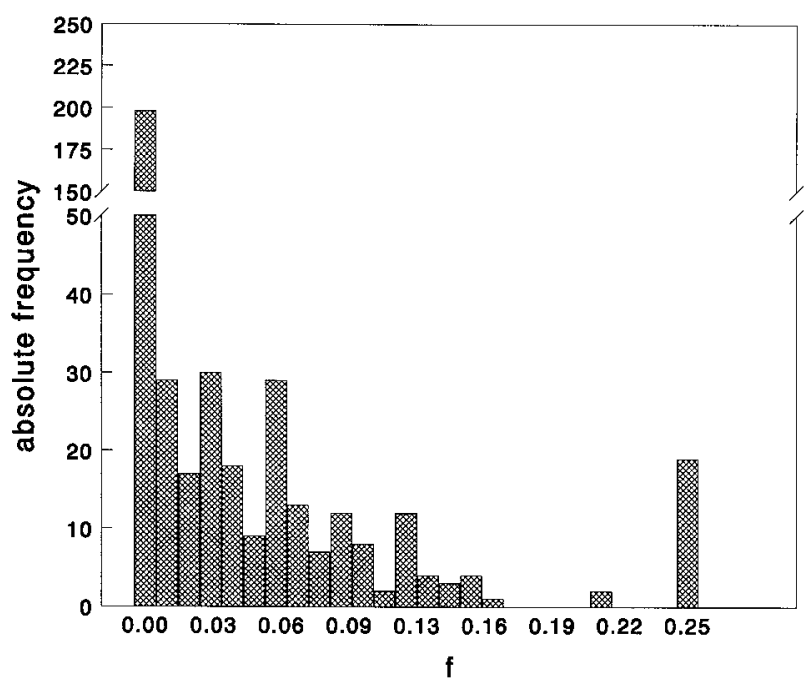

Figure 1: The frequency of inbreeding on Mandarte Island from 1981 to 1995. $f=$ the inbreeding coefficient between the female and the male of a pair. Matings with $f>0.25$ occurred but were grouped with those of $f=0.25$. 

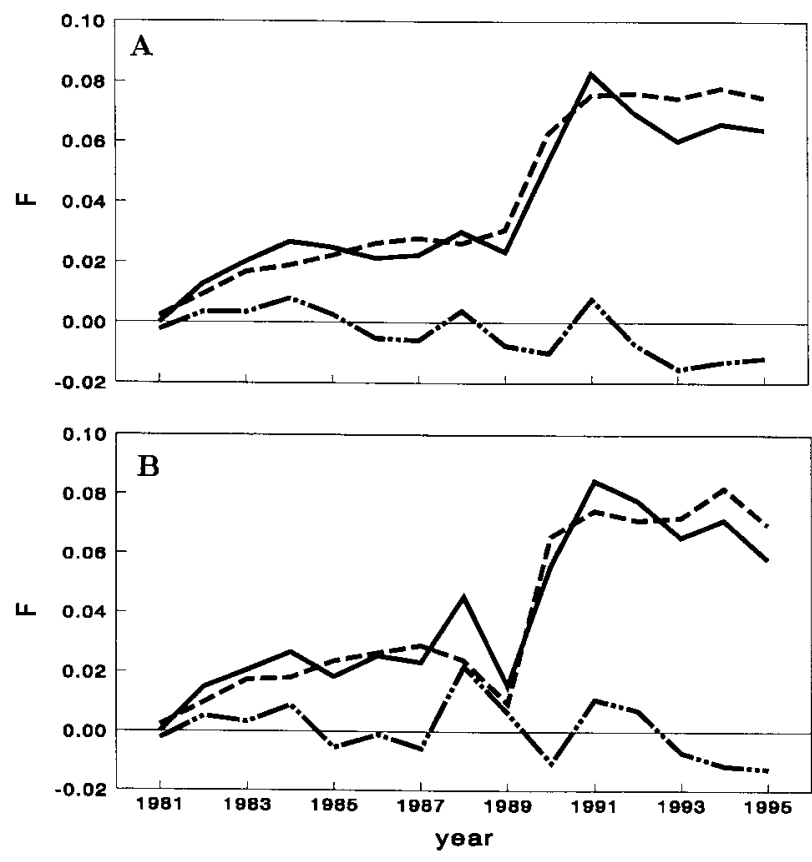

Figure 2: The observed levels of inbreeding $\left(F_{\mathrm{IT}}\right.$, solid line) over the study period and those expected under two scenarios of random mating $\left(F_{\mathrm{ST}}\right.$, broken line). Also given are the corresponding values of nonrandom mating ( $F_{\mathrm{IS}}$, broken-dotted line). An $F_{\mathrm{IS}}$ of 0 indicates that mating was random; $F_{\mathrm{IS}}<0$ indicates inbreeding avoidance. $A$, All breeding birds in the population were assumed to be available as potential mates (All Mates). $B$, Only birds who were not previously mated to the same partner were assumed to be available as potential mates (New Mates). A sharp increase in inbreeding after 1989 occurred coincident with a bottleneck that reduced the population to $6 \%$ of its previous size (Keller et al. 1994).

\section{Inbreeding Avoidance}

We found no evidence of inbreeding avoidance by mate choice based on any of the three different sets of potential mates. For both sets of available mates (All Mates and New Mates), values were very small and fluctuated around 0 , indicating that there was no preference or avoidance of relatives as mates (fig. 2). In no year was there a statistically significant difference in the mean or the general shape of the expected and observed distributions (table 1). In only $9 \mathrm{yr}$ for All Mates and $8 \mathrm{yr}$ for New Mates, out of the $15 \mathrm{yr}$ of the study, was the observed mean inbreeding lower than the one expected under random mating. Furthermore, closely inbred matings $(f \geq 0.125)$ were as frequent as expected in all years (table 2).

A comparison of the relatedness between neighbors also suggested that, on average, there was neither avoidance nor preference of relatives as mates. There was no significant difference between the relatedness of the pair and the neighbors (table 3). Negative mean differences imply inbreeding avoidance, but only six of the 15 yearly means were negative. We also compared the proportion of close inbreeding ( $f \geq 0.125$ ) observed among the actual pairs with that expected if they had mated with their nearest neighbor. Of all the actual matings, $7.2 \%$ were among close relatives while the corresponding figure for neighbors was $6.9 \%\left(\chi^{2}=0.04, P>.83, N=1,390\right)$.

When analyzing tables of statistical tests it is important to control the tablewide Type I error rate (Rice 1989). We chose to do so by applying Bonferroni corrections. A different approach is to view the tests for each year as multiple tests of the same hypothesis and to use Fisher's combined probability test (Fisher 1958) to evaluate overall significance. This approach also supported our conclusion of no inbreeding avoidance by mate choice: none of the analyses (tables 1-3) showed overall significance ( $P \geq .28$ in all cases).

When tests fail to reject the null hypothesis it is important to make sure that this is most likely because the alternative hypothesis is false and not because of confounding factors or the lack of statistical power. We address these issues in the following.

In an insular population one might expect that unrelated or distantly related mating partners are rare. Thus, observed levels of inbreeding may be close to those expected under random mating even in the presence of inbreeding avoidance mechanisms because individuals are unable to avoid all but the highest levels of inbreeding. Although Mandarte is an island, the variance in inbreeding coefficients is substantial (table 1), partly because of low levels of immigration (on average, 1.18 breeding birds/yr). Hence, unrelated or distantly related mating partners are common, and inbreeding avoidance could have resulted in much lower levels of inbreeding. In 1991, for example, inbreeding avoidance could have led to a complete absence of matings between close relatives and a mean inbreeding coefficient of 0.027 or lower. However, random mating in that year would have led to an average inbreeding coefficient of 0.076 (All Mates, table 1 ) and $25.9 \%$ closely inbred matings (table 2 ), a difference that would have been highly significant $(G=$ $16.69, P=.001)$. Thus, the lack of a significant difference between observed and expected levels of inbreeding is not attributable to a lack of unrelated or distantly related potential mates.

Another scenario that could explain the observed patterns would be that kin recognition is based on familiarity (a common finding in animals; Pusey and Wolf 1996) and that inbreeding avoidance does occur between familiar relatives (siblings raised in the same brood, parents and their offspring) but not between nonfamiliar relatives (siblings raised in different broods). If there is a rel- 
Table 1: The yearly mean, standard deviation, and sample size of the inbreeding coefficients among observed pairs and among pairs had they mated at random

\begin{tabular}{|c|c|c|c|c|c|c|c|c|}
\hline \multirow[b]{2}{*}{ Year } & \multicolumn{3}{|c|}{$\begin{array}{l}\text { Inbreeding of observed } \\
\text { pairs }\end{array}$} & \multicolumn{3}{|c|}{$\begin{array}{l}\text { Inbreeding of random } \\
\text { pairs }\end{array}$} & \multicolumn{2}{|c|}{$\mathrm{P}$} \\
\hline & $\bar{X}$ & $S D$ & $\mathrm{~N}$ & $\overline{\mathrm{X}}$ & $S D$ & $\mathrm{~N}$ & U-test & $K-S$ \\
\hline \multicolumn{9}{|c|}{ All Mates: } \\
\hline 1981 & 0 & 0 & 20 & .0023 & .0130 & 360 & .39 & .99 \\
\hline 1982 & .0127 & .0492 & 27 & .0093 & .0425 & 702 & .55 & .99 \\
\hline 1983 & .0202 & .0565 & 62 & .0168 & .0511 & 3,132 & .37 & .99 \\
\hline 1984 & .0269 & .0686 & 60 & .0190 & .0526 & 3,127 & .87 & .99 \\
\hline 1985 & .0249 & .0602 & 78 & .0223 & .0524 & 5,254 & .95 & .99 \\
\hline 1986 & .0214 & .0423 & 70 & .0264 & .0525 & 3,904 & .91 & .99 \\
\hline 1987 & .0225 & .0360 & 63 & .0282 & .0511 & 3,538 & .86 & .98 \\
\hline 1988 & .0303 & .0611 & 56 & .0264 & .0467 & 2,385 & .90 & .96 \\
\hline 1989 & .0236 & .0195 & 4 & .0310 & .0664 & 16 & .62 & .99 \\
\hline 1990 & .0535 & .0811 & 13 & .0629 & .0958 & 110 & .70 & .91 \\
\hline 1991 & .0828 & .0972 & 31 & .0755 & .0817 & 700 & .97 & .63 \\
\hline 1992 & .0696 & .0815 & 49 & .0762 & .0730 & 1,763 & .20 & .38 \\
\hline 1993 & .0603 & .0514 & 53 & .0745 & .0654 & 2,016 & .16 & .17 \\
\hline 1994 & .0660 & .0407 & 61 & .0780 & .0577 & 3,016 & .20 & .30 \\
\hline 1995 & .0640 & .0358 & 48 & .0748 & .0526 & 1,968 & .33 & .67 \\
\hline \multicolumn{9}{|c|}{ New Mates: } \\
\hline 1981 & 0 & 0 & 19 & .0022 & .0134 & 323 & .42 & .99 \\
\hline 1982 & .0149 & .0532 & 23 & .0098 & .0433 & 506 & .47 & .99 \\
\hline 1983 & .0206 & .0545 & 47 & .0174 & .0479 & 1,677 & .56 & .99 \\
\hline 1984 & .0267 & .0653 & 40 & .0181 & .0495 & 1,400 & .87 & .99 \\
\hline 1985 & .0184 & .0438 & 50 & .0237 & .0506 & 2,256 & .50 & .97 \\
\hline 1986 & .0254 & .0468 & 47 & .0264 & .0502 & 1,760 & .57 & .82 \\
\hline 1987 & .0233 & .0332 & 38 & .0289 & .0103 & 1,332 & .64 & .86 \\
\hline 1988 & .0454 & .0792 & 30 & .0240 & .0451 & 720 & .21 & .56 \\
\hline 1989 & .0155 & .0134 & 3 & .0094 & .0114 & 9 & .55 & .96 \\
\hline 1990 & .0560 & .0842 & 12 & .0659 & .0975 & 99 & .83 & .98 \\
\hline 1991 & .0846 & .0984 & 30 & .0746 & .0812 & 648 & .95 & .50 \\
\hline 1992 & .0779 & .0838 & 32 & .0713 & .0671 & 729 & .97 & .96 \\
\hline 1993 & .0656 & .0497 & 38 & .0722 & .0605 & 1,188 & .69 & .86 \\
\hline 1994 & .0714 & .0340 & 42 & .0820 & .0522 & 1,443 & .37 & .62 \\
\hline 1995 & .0581 & .0385 & 28 & .0699 & .0524 & 700 & .27 & .27 \\
\hline
\end{tabular}

Note: Significance levels of the comparison within each year of the two distributions with a MannWhitney $U$-test $(U$-test) and a Kolmogorov-Smirnov test (K-S) are given. Significant differences are indicated by $P$ values $\leq .0033$ (see "Methods"). All mates: all breeding birds are assumed to be available mates; New Mates: previously mated pairs are excluded from the set of available mates.

atively large proportion of nonfamiliar relatives in the population, this scenario could lead to the apparent absence of inbreeding avoidance. We therefore compared the observed and expected occurrence of matings between siblings raised in the same nest, between siblings raised in different nests, and between parents and their offspring (table 4). Because of the rarity of those matings, data from all years were combined. There was no significant difference between the observed and expected frequencies of sib matings that were between siblings raised in the same nest. In fact, contrary to the proposed scenario, matings between siblings raised in the same nest were observed slightly more often than expected. Parent-offspring matings occurred significantly less often than expected based on matings among All Mates. However, if only New Mates were considered, parentoffspring matings were not significantly less common than expected. Removing all previously mated pairs from the set of available mates generally resulted in a lower expected frequency of close inbreeding (tables 1,2, and 4). This was due to the fact that older birds with several of their offspring in the breeding population often re- 


\section{The American Naturalist}

Table 2: The percentage of all matings that occurred among close relatives $(f \geq$ $0.125)$, as observed each year and as expected each year under two random mating models

\begin{tabular}{lcrlcccc}
\hline & \multicolumn{2}{c}{ All Mates } & & \multicolumn{2}{c}{ New Mates } & \\
\cline { 2 - 3 } Year & Observed & Random & & $\mathrm{P}$ & Observed & Random & $\mathrm{P}$ \\
\hline 1981 & 0 & .6 & .64 & 0 & .6 & .63 \\
1982 & 3.7 & 3.1 & .87 & 4.4 & 3.4 & .81 \\
1983 & 6.5 & 6.7 & .93 & 6.4 & 6.3 & .97 \\
1984 & 11.7 & 7.8 & .30 & 12.5 & 6.7 & .20 \\
1985 & 8.9 & 8.1 & .81 & 5.9 & 7.3 & .70 \\
1986 & 5.6 & 7.8 & .49 & 6.3 & 6.8 & .88 \\
1987 & 4.6 & 6.9 & .45 & 2.6 & 5.6 & .36 \\
1988 & 7.1 & 5.7 & .67 & 13.3 & 5.8 & .14 \\
1989 & 0 & 6.3 & .50 & 0 & 0 &. \\
1990 & 23.1 & 22.7 & .98 & 25.0 & 24.2 & .95 \\
1991 & 29.0 & 25.9 & .70 & 30.0 & 24.9 & .53 \\
1992 & 16.3 & 24.7 & .16 & 15.6 & 22.0 & .38 \\
1993 & 7.6 & 18.7 & .02 & 7.9 & 14.5 & .22 \\
1994 & 6.6 & 15.2 & .04 & 7.1 & 14.2 & .16 \\
1995 & 4.17 & 12.3 & .05 & 7.1 & 9.7 & .64 \\
\hline
\end{tabular}

Note: Sample sizes as given in table 1. $P$ refers to the significance levels from $G$-tests. Significant differences are indicated by $P$ values $\leq .0033$ (see "Methods"). Data are given for the two sets of potentially available mates.

mained paired to the same mate. Removing these already mated pairs from the set of available mates reduced the expected occurrence of close inbreeding through parentoffspring matings.

Finally, a possible explanation for the failure to detect inbreeding avoidance could be that our analyses lacked statistical power. The observed occurrence of close inbreeding (fig. 1) and the small observed values of $F_{\mathrm{IS}}$ in figure 2 suggest that substantial inbreeding avoidance never occurred. Nevertheless, we performed power analyses to determine what level of inbreeding avoidance we should have been able to detect given our data set and the tests we employed in tables 1-3. If sparrows avoided only those matings that would have resulted in very high inbreeding (i.e., matings between full sibs or between parents and their offspring, $f \geq 0.25$ ), our power was low to moderate $(0.2 \%$ for table $1,56.0 \%$ for table 2 , and $28.8 \%$ for table 3 ). However, if song sparrows avoided matings that would have resulted in close inbreeding (i.e., the matings mentioned above and any matings between half-sibs, aunt-nephew, or more highly related birds, $f \geq 0.125$ ) our power to detect inbreeding avoidance was very high $(55.1 \%$ for the results presented in table $1,97.8 \%$ for table 2 , and $95.6 \%$ for table 3 ).

\section{Discussion}

Song sparrows on Mandarte Island mated with relatives as often as expected if matings occur randomly with re-
Table 3: The yearly mean of the differences between the inbreeding coefficient of the observed pair and the coefficients created by artificially pairing the birds with their nearest neighbors

\begin{tabular}{lclll}
\hline Year & Mean difference & SD & $\mathrm{N}$ & $\mathrm{P}$ \\
\hline 1981 & -.002 & .007 & 20 & .99 \\
1982 & .010 & .049 & 27 & .25 \\
1983 & .002 & .055 & 62 & .97 \\
1984 & .010 & .070 & 60 & .91 \\
1985 & .0004 & .061 & 78 & .19 \\
1986 & .002 & .053 & 70 & .82 \\
1987 & .005 & .038 & 63 & .90 \\
1988 & .004 & .067 & 56 & .41 \\
1989 & .007 & .016 & 4 & .5 \\
1990 & -.017 & .071 & 13 & .41 \\
1991 & -.007 & .095 & 31 & .39 \\
1992 & .005 & .079 & 49 & .53 \\
1993 & -.007 & .054 & 53 & .15 \\
1994 & -.011 & .044 & 61 & .04 \\
1995 & -.013 & .039 & 48 & .02 \\
$\bar{X}$ & -.0007 & .0015 & 15 & .85
\end{tabular}

Note: The lack of a difference in the mean relatedness of observed pairs and neighbors indicates that there is no evidence of either avoidance or preference of relatives as mates. The significance levels $(P)$ of Wilcoxon signed-rank tests testing the hypothesis that the differences did not differ from 0 in each year are presented. Significant differences are indicated by $P$ values $\leq .0033$ for the yearly comparisons and by $P$ $\leq .05$ for the analysis of the yearly means. See "Methods" for details. 
Table 4: The observed and expected frequency of all sib matings that were between siblings raised in the same nest and the observed and expected frequency of parent-offspring matings among all matings

\begin{tabular}{|c|c|c|c|c|c|c|c|c|c|}
\hline & \multirow{2}{*}{$\begin{array}{l}\text { Number of } \\
\text { sib matings }\end{array}$} & \multirow{2}{*}{$\begin{array}{l}\% \text { between } \\
\text { siblings from } \\
\text { same nest }\end{array}$} & \multicolumn{2}{|c|}{ Sib matings } & \multirow{2}{*}{$\begin{array}{c}\text { Number of } \\
\text { parent-offspring } \\
\text { matings }\end{array}$} & \multirow{2}{*}{$\begin{array}{c}\text { Total } \\
\text { number of } \\
\text { matings }\end{array}$} & \multirow{2}{*}{$\begin{array}{l}\% \text { parent- } \\
\text { offspring } \\
\text { matings }\end{array}$} & \multicolumn{2}{|c|}{$\begin{array}{c}\text { Parent-offspring } \\
\text { matings }\end{array}$} \\
\hline & & & G & $\mathrm{P}$ value & & & & G & $\mathrm{P}$ value \\
\hline Observed & 16 & 37.5 & $\cdots$ & $\cdots$ & 2 & 479 & .42 & $\cdots$ & $\cdots$ \\
\hline \multicolumn{10}{|l|}{ Expected: } \\
\hline All Mates & 270 & 24.8 & 1.18 & .277 & 307 & 19,977 & 1.54 & 5.48 & .019 \\
\hline New Mates & 218 & 29.4 & .453 & .501 & 137 & 13,051 & 1.05 & 2.32 & .128 \\
\hline
\end{tabular}

Note: Expected frequencies were calculated for both All Mates and New Mates. The results of $G$-tests comparing the observed and expected frequencies are given for each set of potential mates.

spect to inbreeding. Thus, our results do not support the hypothesis that mate choice generally leads to avoidance of inbreeding in birds. The fact that three different assumptions about the sets of potential mates gave similar results suggests that our results are robust. In particular, a generally good correspondence between our results obtained under the assumption that matings occurred at random in the population and by the nearest neighbor method indicates that the observed pattern of dispersal had no effects on observed levels of inbreeding (see also Arcese 1989b). Thus, finite population size rather than differential dispersal or inbreeding avoidance behaviors seems to be the key factor affecting annual levels of inbreeding on Mandarte Island.

While matings between familiar siblings were as frequent as expected, parent-offspring matings occurred significantly less often than expected under one null model (All Mates) but not under the other (New Mates). Thus, we cannot exclude a slight tendency to avoid parentoffspring matings, but the conflicting results from the two different null models preclude a firm conclusion. The reduced incidence of observed parent-offspring matings as compared with the All Mates null model may simply indicate that the assumption that previously mated pairs are all available for remating is incorrect.

Overall, our inability to reject the null hypothesis of random mating was unlikely to have resulted from low statistical power. Our analyses suggest that we had ample statistical power to reject the hypothesis of no inbreeding avoidance at the $f \geq 0.125$ level. Statistical power was lower for less pronounced inbreeding avoidance (no matings with $f \geq 0.25$ ). Low statistical power was partly the result of conservative significance levels $(P \leq .0033)$. None of the individual comparisons was significant at the $P \leq .01$ level, and at this significance level, our analyses reached a power of $69.6 \%$ for rejecting the hypothesis of no inbreeding avoidance at $f \geq 0.25$. Overall, because we used three different approaches to test the null hypothesis of no inbreeding avoidance and because we achieved reasonable power in each single case, low statistical power is unlikely to have prevented our detection of inbreeding avoidance among song sparrows on Mandarte Island. However, our power calculations demonstrate that a large percentage of expected matings between siblings or parents and their offspring would be required to have high confidence (e.g., a power of 95\%) in the presence or absence of inbreeding avoidance at the $f=0.25$ level. Few natural populations will meet this criterion.

Our results support most other published studies of inbreeding avoidance by mate choice in wild birds. Inbreeding avoidance was not detected in great tits (Parus major) on Vlieland, the Netherlands (van Noordwijk et al. 1985; van Tienderen and van Noordwijk 1988), or in great tits in Wytham Wood, United Kingdom (Bulmer 1973; Greenwood et al. 1978). Medium ground finches (Geospiza fortis) on Daphne Major in the Galápagos, Ecuador (Gibbs and Grant 1989), and collared flycatchers (Ficedula albicollis) on the Swedish island of Gotland (Pärt 1996) also failed to show a deviation from the pattern expected under random mating. In contrast, inbreeding avoidance was observed in the cooperatively breeding acorn woodpecker (Melanerpes formicivorus; Koenig and Pitelka 1979; Koenig et al. 1998). In acorn woodpeckers, inbreeding is avoided by groups of siblings of the same sex dispersing together and by the failure of nondispersing offspring to reproduce.

\section{Why Do Song Sparrows Not Avoid Inbreeding?}

It is curious that song sparrows on Mandarte Island mate with moderate to close relatives despite substantial reductions in fitness that occur as a consequence of inbreeding (Keller et al. 1994; Keller 1998). However, inbreeding avoidance should evolve only when the costs of inbreeding exceed those of avoiding inbreeding (Waser et al. 1986). Costs of inbreeding avoidance include those of dispersal and those of forfeiting outbred matings by choosing to inbreed. Kin recognition should be the 


\section{The American Naturalist}

cheapest inbreeding avoidance mechanism (Blouin and Bouin 1988), because the costs of dispersal can be high (Johnson and Gaines 1990). Thus, the absence of inbreeding avoidance on Mandarte Island suggests either that song sparrows are unable to recognize kin or that the costs of inbreeding avoidance by mate choice are higher than those of inbreeding. We will discuss these two alternatives in turn. There are few data on kin recognition (either based on familiarity or other mechanisms) and its effects on mating patterns in birds. Some studies in captivity (e.g., Bateson 1982; Burley et al. 1990) and in the wild (e.g., McGregor and Krebs 1982; Grant 1984) have shown that it can occur. However, it is currently unclear how widespread the ability to recognize kin is in birds. The majority of the studies on kin recognition, inbreeding avoidance, and mate choice have focused on small mammals and primates (Blouin and Blouin 1988; Pusey and Wolf 1996), taxa that have a well-developed sense of smell that may be used in kin recognition. The mechanisms of kin recognition in birds are mostly unknown. An obvious mechanism would be song as shown, for example, for zebra finches (Taeniopygia guttata; Miller 1979) and large ground finches (Geospiza magnirostris; Grant 1984). In song sparrows, however, young generally do not learn their fathers' songs (Nice 1943). In fact, none of the 21 males who were studied in depth on Mandarte shared any song types with his father (Cassidy 1993). Hence, song does not provide a mechanism for kin recognition in song sparrows. Furthermore, our results for the relative frequency of matings between familiar siblings (raised in the same nest) versus unfamiliar siblings (raised in different nests; table 4) suggest that familiarity is not used as a rule of thumb to determine relatedness.

Even if mechanisms to recognize kin exist (such as familiarity, e.g.) kin recognition systems and inbreeding avoidance may still not evolve. Inbreeding may not occur often enough in the species as a whole to exert enough selection pressure to lead to the evolution of kin recognition systems. The song sparrow is a widely distributed species on the North American continent, and most mainland song sparrow populations are much larger and less isolated than that on Mandarte Island (see, e.g., Smith et al. 1996). Thus, the occurrence of inbreeding in most song sparrow populations is likely lower than on Mandarte and, thus, perhaps not high enough to lead to the evolution of avoidance behaviors. However, although close inbreeding has been reported in mainland song sparrow populations (Nice 1943), sufficient data on the levels of inbreeding in mainland versus island populations are lacking to address this hypothesis empirically.

An alternative explanation for the lack of inbreeding avoidance in song sparrows is that the fitness costs of avoiding inbreeding are higher than those associated with inbreeding. Waser et al. (1986) published a general model to investigate the conditions under which individuals should avoid inbreeding and the conditions under which they should tolerate inbreeding. Their model suggests that in most cases the sole factor determining whether inbreeding avoidance or tolerance spreads is the costs of inbreeding avoidance relative to the costs of inbreeding. An important component of the latter are the number of outbred matings an individual forgoes by mating with a relative (Waser et al. 1986). Several lines of evidence suggest that the costs of avoiding inbred matings might be substantial on Mandarte, especially for males. First, the sex ratio of territorial birds favors males in most years $(\bar{X}=0.92$, range $=0.64-1.07$; Arcese $1989 a$ ), and up to $28 \%$ of males exist as nonterritorial floaters (Smith and Arcese 1989). Females also compete for breeding territories, and some females settle long after other females have initiated breeding. Thus, territory acquisition is a highly competitive process for both sexes (Arcese 1989a, 1989b). It is possible, therefore, that choosing not to settle on an available territory when this would result in inbreeding is costly because it could lead to breeding later, breeding in a territory of lower quality, or not breeding at all. Further work to quantify the costs of inbreeding avoidance by song sparrows on Mandarte Island is needed to determine if inbreeding avoidance is likely to evolve.

\section{What Causes the Variation in Inbreeding Avoidance among Bird Species?}

It is a puzzle that a species like the acorn woodpecker displays such strong tendencies to avoid inbreeding (Koenig and Pitelka 1979; Koenig et al. 1998), while none of the other bird species studied in detail showed evidence of inbreeding avoidance by mate choice. In order to explore potential explanations for these differences we employed a simple model, which we present in detail in the appendix.

In brief, we asked if an individual that forgoes breeding in its first year in order to avoid inbreeding, but as a result will breed in all following years with an unrelated mate, will achieve lifetime reproductive success (LRS) similar to that achieved by an identical individual that breeds with a relative in its first and all later breeding seasons. We assumed that birds not avoiding inbreeding mate with a very close relative $(f=0.25)$ in the first and all subsequent years. Lifetime reproductive success was then calculated for individuals that tolerated inbreeding and those that avoided it. The fitness costs of inbreeding were incorporated by reducing the fecundity of inbred matings by a multiplicative factor. Model parameters 


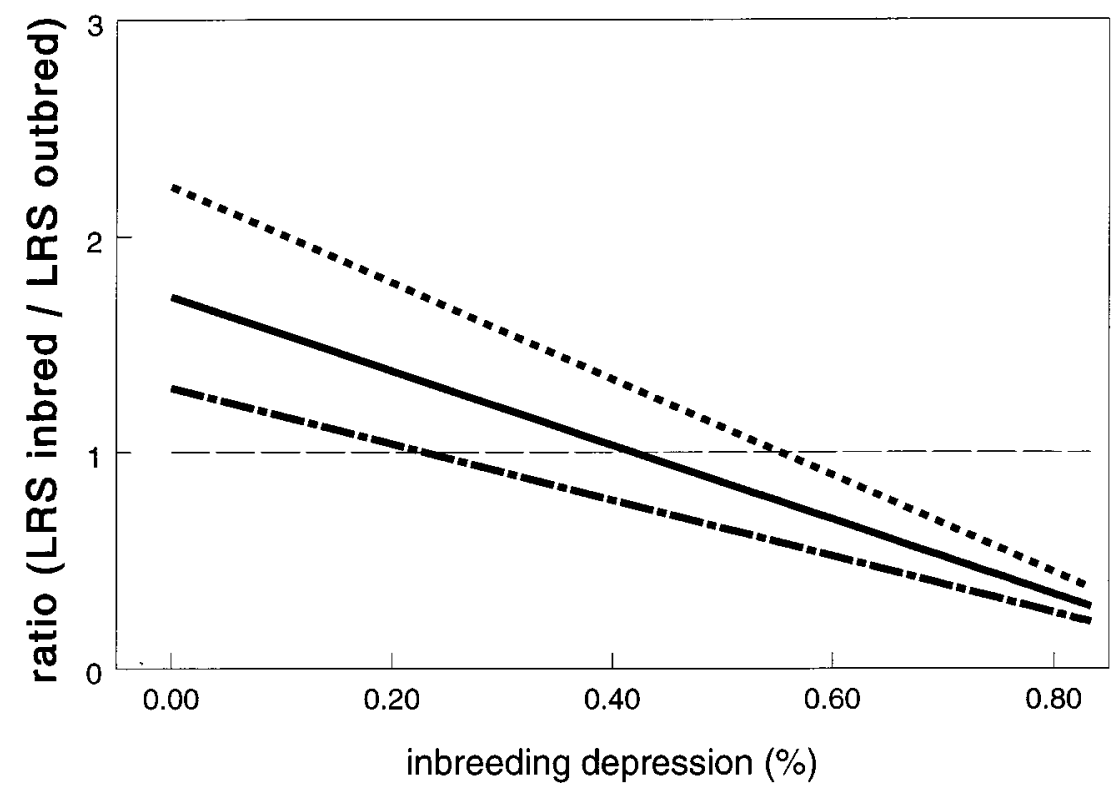

Figure 3: The ratio of lifetime reproductive success expected by "inbred" and "outbred" mating strategies as a function of the severity of inbreeding depression (see text and appendix). Model outputs are shown based on parameters derived from the literature for song sparrows (solid line), great tits (dotted line), and acorn woodpeckers (dashed line).

were taken from empirical observations from Mandarte song sparrows, acorn woodpeckers (Koenig and Mumme 1987), and great tits (McCleery and Perrins 1989).

Inbreeding avoidance is favored where the LRS of individuals tolerating inbreeding falls below that of individuals avoiding it, that is, where the ratio of LRS falls below 1 (fig. 3). The results of our simple model (see appendix for parameters) suggest that this ratio falls below 1 when inbred matings experience a reduction in reproductive success of $42 \%$ in song sparrows and $55 \%$ in great tits. For acorn woodpeckers, our comparison suggests that inbreeding avoidance becomes advantageous for values of inbreeding depression above 22\%. Thus, inbreeding avoidance is advantageous for acorn woodpeckers at levels of inbreeding depression of approximately half those of song sparrows. The costs of more distant inbreeding than what we assumed here are lower, and, thus, higher levels of inbreeding depression would be necessary to favor avoidance of more distant inbreeding.

The differences in our model among species in the benefits of inbreeding avoidance are due mostly to differences in survival. Acorn woodpeckers are much more likely to survive to settle with a nonrelative in a following year than are song sparrows or great tits. Hence, for acorn woodpeckers inbreeding avoidance may result in higher LRS even when the birds forgo breeding for an entire season to obtain an outbred mating.

Repeated runs of the model with various parameter combinations showed that the outcome was most sensi- tive to variation in survival. Varying the age-dependent fecundities affected the results to a much lesser degree, and variation in the maximum reproductive life span (while holding survival probabilities constant) had very minor effects.

The model we presented here is simple and makes various simplifying assumptions. For example, the costs of inbreeding avoidance were assumed to be constant (loss of one breeding season), and no difference in costs between sexes was considered. Moreover, observations in song sparrows show that males that did not breed in their first year did not always settle in the second year (Smith and Arcese 1989) and that birds mated to a firstdegree relative sometimes switch mates later in their life. Nevertheless, our model indicates that annual survival probabilities may explain some of the observed patterns of inbreeding avoidance among bird species; and it suggests that if avoiding inbreeding entails delaying breeding, inbreeding avoidance will be less advantageous and thus less prevalent in short- as opposed to long-lived species.

\section{Acknowledgments}

We thank the many people who worked on Mandarte Island at one time or another and who collected the data presented here, including most recently A. Cassidy, M. Hatch, I. Heaven, W. Hochachka, G. Jongejan, J. Leary, and J. Smith. The Tseycum and Tsawout Indian bands 


\section{The American Naturalist}

kindly allowed us to work on Mandarte Island. J. Crow suggested the use of Wright's $F$ statistics. L. FreemanShade helped with PEDSYS. R. O'Brien made UnifyPow available. Discussions with J. Crow, C. Denniston, L. Fishman, D. Heisey, W. Hochachka, A. Ives, W. Koenig, D. O’Brien, K. Petren, J. Smith, S. Stearns, D. Waller, and an anonymous reviewer greatly improved this manuscript and our understanding of the subject. J. Smith and S. Stearns provided crucial help during all stages of this project. Funding provided by the Priority Programme Environment/Biodiversity of the Swiss National Science Foundation (5000-034715 to S. Stearns), the Roche Research Foundation (Switzerland), and the National Science Foundation (IBN 9458122) is gratefully acknowledged.

\section{APPENDIX}

\section{Costs of Inbreeding and Inbreeding Avoidance}

To model the costs of an individual's mating decisions, we followed the approach proposed by Sutherland (1996, p. 73). Lifetime reproductive success (LRS) of an individual was calculated as:

$$
\text { LRS }=S^{b-1} \sum_{x=b}^{n} S^{x} F_{x} d,
$$

where $x$ is age, $S$ is the age-independent annual survival rate, $F_{x}$ are the age-dependent fecundities, $b$ is the age at first breeding, $n$ is the maximum reproductive life span, and $d$ is the inbreeding depression. Inbreeding depres- sion was incorporated in the model by reducing the fecundities by a multiplicative factor. Hence, the model allowed us to calculate the ratio of the LRS of an individual that tolerated inbreeding and the LRS of an individual that avoided inbreeding.

We parameterized our model for the Mandarte song sparrows, Wytham Wood great tits, and Hastings Reservation acorn woodpeckers. Age-independent survival rates were estimated as the geometric mean of annual survival rates averaged over both sexes. Table A1 presents the parameters used in the model.

\section{Literature Cited}

Arcese, P. 1989a. Intrasexual competition and the mating system in primarily monogamous birds: the case of the song sparrow. Animal Behaviour 38:96-111.

- 1989b. Intrasexual competition, mating system and natal dispersal in song sparrows. Animal Behaviour 38:958-979.

Bateson, P. 1978. Sexual imprinting and optimal outbreeding. Nature (London) 273:659-660.

- 1982. Preferences for cousins in Japanese quail. Nature (London) 295:236-237.

- 1983. Optimal outbreeding. Pages 257-277 in P. Bateson, ed. Mate choice. Cambridge University Press, Cambridge.

Blouin, S. F., and M. Blouin. 1988. Inbreeding avoidance behaviors. Trends in Ecology \& Evolution 3:230-233.

Boyce, A. J. 1983. Computation of inbreeding and kinship coefficients on extended pedigrees. Journal of Heredity 74:400-404.

Table A1: Parameters used in the model

\begin{tabular}{lccc}
\hline Parameter & Song sparrow & Acorn woodpecker & Great tit \\
\hline Annual survival S (geometric mean) & .515 & .776 & .416 \\
Maximum reproductive life span (yr) & 7 & 15 & 8 \\
Age-dependent fecundity: & & & \\
Age 1 & .69 & $*$ & .865 \\
Age 2 & 1.39 & $*$ & 1.139 \\
Age 3 & 1.17 & $*$ & 1.139 \\
Age 4 & 1.11 & $*$ & 1.139 \\
Age 5 & 1 & $*$ & .965 \\
Age 6 & 1 & $*$ & .965 \\
Age 7 & 1 & $*$ & .965 \\
Age 8 & $\ldots$ & $*$ & .965 \\
\hline
\end{tabular}

Note: Geometric mean annual survival rates were calculated directly for the song sparrows. Estimates of all other parameters were taken from the literature. Age-dependent fecundities for the song sparrows were taken from Nol and Smith (1987). All parameters for the acorn woodpeckers are from Koenig and Mumme (1987). Survival rates for the great tits were calculated from Clobert et al. (1988, table 4), and all other parameters for the great tits are from McCleery and Perrins (1988).

* Age independent: 1 . 
Bulmer, M. G. 1973. Inbreeding in the great tit. Heredity 30:313-325.

Burley, N., C. Minor, and C. Strachan. 1990. Social preference of zebra finches for siblings, cousins and nonkin. Animal Behaviour 39:775-784.

Cassidy, A. L. E. V. 1993. Song variation and learning in island populations of song sparrows. Ph.D. diss. University of British Columbia, Vancouver.

Charlesworth, D., and B. Charlesworth. 1987. Inbreeding depression and its evolutionary consequences. Annual Review of Ecology and Systematics 18:237-268.

Chen, X. 1993. Comparison of inbreeding and outbreeding in hermaphroditic Arianta arbustorum (L.) (land snail). Heredity 71:456-461.

Clobert, J., C. M. Perrins, R. H. McCleery, and A. G. Gosler. 1988. Survival rate in the great tit, Parus major, in relation to sex, age, and immigration status. Journal of Animal Ecology 57:287-306.

Crow, J. F., and M. Kimura. 1970. An introduction to population genetics theory. Alpha Editions, Minneapolis.

Darwin, C. 1876. The effects of crossing and self-fertilization in the vegetable kingdom. John Murray, London.

Drent, R., G. F. van Tets, F. Tompa, and K. Vermeer. 1964. The breeding birds of Mandarte Island, British Columbia. Canadian Field-Naturalist 78:208-263.

Fisher, R. A. 1949. The theory of inbreeding. 1st ed. Oliver \& Boyd, Edinburgh.

. 1958. Statistical methods for research workers. 13th ed. Hafner, New York.

Gibbs, H. L., and P. R. Grant. 1989. Inbreeding in Darwin's medium ground finches (Geospiza fortis). Evolution 43:1273-1284.

Grant, B. R. 1984. The significance of song variation in a population of Darwin's finches. Behaviour 89:90-116.

Greenwood, P. J., P. H. Harvey, and C. M. Perrins. 1978. Inbreeding and dispersal in the great tit. Nature (London) 271:52-54.

Hamilton, W. D. 1987. Discriminating nepotism: expectable, common, overlooked. Pages 417-437 in D. J. C. Fletcher and C. D. Michener, eds. Kin recognition in animals. Wiley, Colchester.

Harvey, P. H., and K. Ralls. 1986. Do animals avoid incest? Nature (London) 320:575-576.

Hoogland, J. L. 1992. Levels of inbreeding among prairie dogs. American Naturalist 139:591-602.

Jiménez, J. A., K. A. Hughes, G. Alaks, L. Graham, and R. C. Lacy. 1994. An experimental study of inbreeding depression in a natural habitat. Science (Washington, D.C.) 266:271-273.

Johnson, M. L., and M. S. Gaines. 1990. Evolution of dispersal: theoretical models and empirical tests using birds and mammals. Annual Review of Ecology and Systematics 21:449-480.
Keller, L. F. 1996. Inbreeding in an insular population of song sparrows. Ph.D. diss. University of Wisconsin, Madison.

- 1998. Inbreeding and its fitness effects in an insular population of song sparrows (Melospiza melodia). Evolution 52:240-250.

Keller, L. F., P. Arcese, J. N. M. Smith, W. M. Hochachka, and S. C. Stearns. 1994. Selection against inbred song sparrows during a natural population bottleneck. Nature (London) 372:356-357.

Koenig, W. D., and R. L. Mumme. 1987. Population ecology of the cooperatively breeding acorn woodpecker. Princeton University Press, Princeton, N.J.

Koenig, W. D., and F. A. Pitelka. 1979. Relatedness and inbreeding avoidance: counterploys in the communally nesting acorn woodpecker. Science (Washington, D.C.) 206:1103-1105.

Koenig, W. D., J. Haydock, and M. T. Stanback. 1998. Reproductive roles in the cooperatively breeding acorn woodpecker: incest avoidance versus reproductive competition. American Naturalist 151:243-255.

McCleery, R. H., and C. M. Perrins. 1988. Lifetime reproductive success of the great tit, Parus major. Pages 136-153 in T. H. Clutton-Brock, ed. Reproductive success: studies of individual variation in contrasting breeding systems. University of Chicago Press, Chicago.

McGregor, P. K., and J. R. Krebs. 1982. Mating and song types in the great tit. Nature (London) 297:60-61.

Miller, D. B. 1979. Long-term recognition of father's song by female zebra finches. Nature (London) 280: 389-390.

Nice, M. M. 1943. Studies in the life history of the song sparrow. II. Transactions of the Linnean Society of New York 6:1-329.

Nol, E., and J. N. M. Smith. 1987. Effects of age and breeding experience on seasonal reproductive success in the song sparrow. Journal of Animal Ecology 56: 301-314.

O'Brien, R. G., and K. E. Muller. 1993. Unified power analysis for $t$ tests through multivariate hypotheses. Pages 297-344 in L. K. Edwards, ed. Applied analysis of variance in behavioral science. Dekker, New York.

Pärt, T. 1996. Problems with testing inbreeding avoidance: the case of the collared flycatcher. Evolution 50: 1625-1630.

Pusey, A. E. 1987. Sex-biased dispersal and inbreeding avoidance in birds and mammals. Trends in Ecology \& Evolution 2:295-299.

Pusey, A., and M. Wolf. 1996. Inbreeding avoidance in animals. Trends in Ecology \& Evolution 11:201-206.

Ralls, K., P. H. Harvey, and A. M. Lyles. 1986. Inbreeding in natural populations of birds and mammals. Pages 
392 The American Naturalist

35-56 in M. E. Soulé, ed. Conservation biology: the science of scarcity and diversity. Sinauer, Sunderland, Mass.

Ralls, K., J. D. Ballou, and A. Templeton. 1988. Estimates of lethal equivalents and the cost of inbreeding in mammals. Conservation Biology 2:185-193.

Rice, W. R. 1989. Analyzing tables of statistical tests. Evolution 43:223-225.

SAS Institute. 1990. SAS/STAT user's guide, version 6. 4th ed. SAS Institute, Cary, N.C.

Smith, J. N. M. 1981. Cowbird parasitism, host fitness, and age of the host female in an island song sparrow population. Condor 83:152-161.

Smith, J. N. M., and P. Arcese. 1989. How fit are floaters? consequences of alternative territorial behaviors in a nonmigratory sparrow. American Naturalist 133: 830-845.

Smith, J. N. M., M. J. Taitt, C. M. Rogers, P. Arcese, L. F. Keller, A. L. E. V. Cassidy, and W. M. Hochachka. 1996. A metapopulation approach to the population biology of song sparrows Melospiza melodia. Ibis $138: 120-128$.

Southwest Foundation for Biomedical Research. 1998. PedSys user's manual, version 2.0. Southwest Foundation for Biomedical Research, San Antonio, Tex. http://www.sfbr.org/sfbr/public/software/pedsys/ pedsys.html, March 18.

Sutherland, W. J. 1996. From individual behavior to population ecology. Oxford University Press, Oxford.

Tompa, F. S. 1964. Factors determining numbers of song sparrows Melospiza melodia (Wilson) on Mandarte Island, B.C., Canada. Acta Zoologica Fennica 109:1-73. van Noordwijk, A. J., P. H. van Tienderen, G. de Jong, and J. H. van Balen. 1985. Genealogical evidence for random mating in a natural population of the great tit (Parus major L.). Naturwissenschaften 72:104-105.

van Tienderen, P. H., and A. J. van Noordwijk. 1988. Dispersal, kinship and inbreeding in an island population of the great tit. Journal of Evolutionary Biology 1: $117-138$.

Waser, P. M., S. N. Austad, and B. Keane. 1986. When should animals tolerate inbreeding? American Naturalist 128:529-537.

Weatherhead, P. J., and K. A. Boak. 1986. Site infidelity in song sparrows (Melospiza melodia). Animal Behaviour 34:1299-1310.

Weatherhead, P. J., and M. R. L. Forbes. 1994. Natal philopatry in passerine birds: genetic or ecological influences? Behavioral Ecology 5:426-433.

Wright, S. 1965. The interpretation of population structure by F-statistics with special regard to systems of mating. Evolution 19:395-420.

. 1969. Evolution and the genetics of populations. Vol. 2. The theory of gene frequencies. University of Chicago Press, Chicago.

. 1977. Evolution and the genetics of populations. Vol. 3. Experimental results and evolutionary deductions. University of Chicago Press, Chicago.

Associate Editor: Marlene Zuk 